\title{
Molecular Characterisation of Carcinoma Breast Using Surrogate IHC Markers-ER/PR, HER-2, CK5/6, EGFR, Ki-67
}

\author{
Krishna Govindan ${ }^{1}$, Shinu Ninan ${ }^{2}$ \\ ${ }^{1}$ Department of Pathology, Government Medical College, Trivandrum, Kerala, India. \\ ${ }^{2}$ Department of Pathology, Government Medical College, Trivandrum, Kerala, India.
}

\section{ABSTRACT}

\section{BACKGROUND}

Breast cancer is a heterogenous disease, and this term encompasses a variety of entities with distinct morphological features and clinical behaviour. Even the tumours with the same histological type show remarkably different clinical behaviour. And this was found to be due to the aberrations at the molecular level. The objective of this study is to do molecular characterisation of carcinoma breast using surrogate markers.

\section{METHODS}

This is an Observational Diagnostic Study done in the Department of Pathology, Government Medical College, Thiruvananthapuram, during a period of 1 year and 2 months. The study period was from July 2015 to September 2016. During this period all breast cancer cases were taken fulfilling the inclusion criteria. The surrogate markers used in this study were ER, PR, HER2, CK5/6, EGFR \& Ki-67. Immunohistochemistry was done at our laboratory using standard controls. The IHC stained slide was read using standard protocols.

\section{RESULTS}

There were 1916 various breast specimens received in the department (Government Medical College, Trivandrum). Of this, 558 specimens were included in our study (satisfying inclusion criteria). In our study, we found that Luminal-A was the most predominant type comprising about $43 \%$, followed by Triple Negative and HER2 Positive types which makes $21 \%$. Least dominant was the Luminal-B type which accounts for only $13 \%$ of all breast cancer. The Triple Negative cancers included the basal-like and the Non-Basal-like tumours. Of this $76.85 \%$ showed a basal like phenotype (CK5/6 \& EGFR +ve) and $23.14 \%$ was negative for even basal markers.

\section{CONCLUSIONS}

So, we could conclude that the surrogate markers are efficient enough to be used for subtyping breast carcinoma at molecular level in most institutions for effective "Patient-Tailored Treatment" plans. The panel of 6 markers, as used in our study, has indeed got an upper hand in more accurately and precisely subtyping the "Multifaceted" breast carcinoma.

\section{KEY WORDS}

Breast Cancer Subtypes, Molecular Classification, Surrogate IHC Markers

\author{
Corresponding Author: \\ Dr. Shinu Ninan, \\ T. C. $14 / 542$, Nandavanam, \\ Vikas Bhavan, P. O., \\ Trivandrum-695033, Kerala, India. \\ E-mail: shinuninan7@gmail.com
}

DOI: $10.14260 / \mathrm{jemds} / 2019 / 750$

Financial or Other Competing Interests: None.

How to Cite This Article:

Govindan K, Ninan S. Molecular characterisation of carcinoma breast using surrogate IHC markers-ER/PR, HER-2, CK5/6, EGFR, Ki-67. J. Evolution Med. Dent. Sci. 2019;8(46):3464-3468, DOI: $10.14260 /$ jemds $/ 2019 / 750$

Submission 31-08-2019,

Peer Review 19-10-2019,

Acceptance 26-10-2019,

Published 18-11-2019. 


\section{BACKGROUND}

Breast carcinoma is the most common malignant tumour and the leading cause of carcinoma death in women worldwide. It accounts for $23 \%$ of all cancers in women globally and $27 \%$ in affluent countries. It is more than twice as common as cancer at any other site. In the management of patients with breast disease, close cooperation between surgeon, medical oncologist, radiation oncologist, radiologist, and pathologist is always of the utmost importance. A crucial development in the treatment of breast carcinoma has been the realization that the presence of hormone (Oestrogen and progesterone) receptors in the tumour tissue correlates well with response to hormone therapy and chemotherapy. As a matter of fact, oestrogen receptor status is regarded at present as the most powerful predictive marker in breast cancer management.

Breast cancer was traditionally perceived as a single disease; however, recent advances in gene expression and genomic profiling have revealed that breast cancer is in fact a collection of diseases exhibiting distinct anatomical features, responses to treatment and survival outcomes. Consequently, a number of schemes have been proposed for subtyping of breast cancer to bring out the biological and clinically relevant characteristics of the subtypes. Although some of these schemes capture underlying molecular differences, others predict variations in response to treatment and survival patterns. However, despite this diversity in the approaches, it is clear that molecular mechanisms drive clinical outcomes, and therefore an effective scheme should integrate molecular as well as clinical parameters to enable deeper understanding of cancer mechanisms and allow better decision making in the clinic.

Studies of breast cancers using gene expression profiling have identified several major breast cancer subtypes beyond the traditional "Hormone receptor negative and positive" types. The most reproducibly identified molecular subtypes among the Hormone receptor positive cancers are the LuminalA \& Luminal-B groups. The HER2 \& basal like are the major molecular subtypes identified among Hormone receptor negative breast cancers. Other molecular subtypes such as Luminal C \& normal breast like groups have also been identified in some studies but are less well characterised than Luminal-A, Luminal-B, HER2 \& Basal Like. These breast cancer molecular subtypes differ with regard to their patterns of gene expression, clinical features, response to treatment and prognosis. This sub typing is done with surrogate IHC markersKi-67, CK5/6, EGFR. So, to classify breast cancer on molecular level we need 6 surrogate markers namely ER, PR, HER2 \& the earlier mentioned 3 IHC.

It is clear that the role of IHC in detecting biomarker expression in pathology largely depends on research studies which demonstrate differential immunohistochemical expression and other studies that show good correlation between positive expression and response to new therapy. Thus, by combining a panel of markers we can correctly arrive at a accurate diagnosis. Earlier there was a panel of 3 markers used to classify breast cancer. But later studies revealed that it was insufficient for accurately pinpointing the subtype of breast cancer. So now the most accepted IHC marker is a panel of 6 markers. With this a more efficient subtyping can be done which is more beneficial for the patient for the planning of "patient-tailored" treatment.

\begin{tabular}{|c|c|}
\hline Molecular Subtypes & Biomarker Profile \\
\hline Luminal-A & ER+ and/or PR+, HER2-, low ki-67 (<14\%) \\
\hline Luminal-B & ER+ and/or PR+, HER2-, high ki-67 (>14\%) \\
\hline HER2 & ER-,PR-, and HER2+ \\
\hline Basal-Like & ER-, PR-, HER2- and CK5/6 \&/or EGFR+ \\
\hline \multicolumn{2}{|c|}{ Table 1. Subtyping Carcinoma Breast with IHC } \\
\hline $\begin{array}{l}\text { By using the panel of 6 markers, the above defined criteria is made use for the } \\
\text { subtyping of breast cancer. }\end{array}$ \\
\hline
\end{tabular}

\section{METHODS}

This is a descriptive study. All cases of carcinoma breast from TruCut biopsies, MRM, Lumpectomies, Mastectomies and other surgical procedures (Like BCS, Wedge biopsy etc.) done in Government Medical College Trivandrum and diagnosed by histopathological methods in the Department of Pathology, Government Medical College, Trivandrum. The study was approved by Institute of Ethical Committee. The study duration was six months.

\section{Inclusion Criteria}

1. Histopathologically proven cases of Carcinoma Breast whose surgeries were done in Government Medical College, Trivandrum.

2. Those specimens that are put in the special fixative which is supplied to the Operation Theatre by investigator.

\section{Exclusion Criteria}

1. Post-chemotherapy cases.

2. Specimen which does not have adequate tissue.

\section{Statistical Analysis}

The Statistical Package for Social Science \{SPSS $\}$ Version 20 will be used for Data Analysis. Mean, median, and SD are used to describe quantitative data. Qualitative data are summarized using frequency and percentage.

\section{RESULTS}

There was a total of 1916 various breast specimen that came to the department during July 2015 to September 2016 time. Of it 1154 turned out to be benign and 762 turned to be malignant. Since our population of interest is carcinoma breast, the 762 malignant cases were taken into consideration. Of this, 109 malignant cases were from outside hospitals (General hospital, ESI hospital, Neyyatinkara hospital etc.) which did not satisfy the inclusion criteria. The rest 653 malignant cases were from Government Medical College Trivandrum which was treated by neutral buffered formalin supplied to the theatre from pathology department. This included MRM specimen, Lumpectomy, TruCut \& Mastectomy specimens. Among this 96 sample could not be included in the study due to reasons of tissue insufficiency, or blocks were taken immediately after a histopathological diagnosis of carcinoma breast, or post chemotherapy cases, or completion MRM done in a previously diagnosed breast cancer. Excluding all this, the final study consisted of 557 various breast specimens diagnosed as 
carcinoma breast and the full panel of IHC could be done with final subtyping.

\section{Male Breast Cancer and its Age-Incidence}

Global International trend of occurrence of Breast cancer in male comprises of less than $1 \%$ of all breast cancer cases 1 . Because of its rarity, comparatively little is known about its age specificity, aetiology and types. ${ }^{1,2}$ The female to male incidence rate ratios (FMIRR) have been calculated across countries and found to be 122 in a retrospective study by Diana et al. ${ }^{2}$ In our study of 557 cases, we got 9 male breast cancer cases and 548 female breast cancer cases.

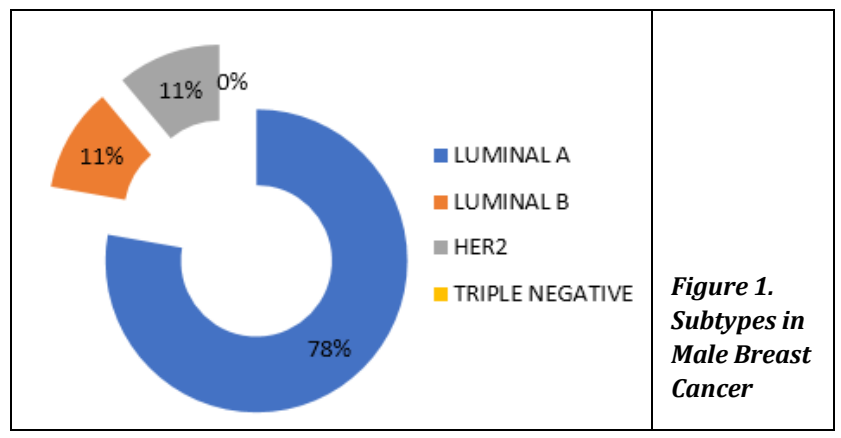

\section{Female Breast Cancer Age-Incidence Curve}

The lowest age in our study was 46 years and the highest age 85 years with respect to male breast cancer cases. This was seen to be higher when compared to female breast cancer group where the lowest age was 26 years and highest age 85 years. This brings the mean age in female breast cancer as 54 years which is a younger mean age as compared to male breast cancer which is 65 years. As with most epithelial tumours, the incidence of breast cancer increases rapidly with age. 3,4 The incidence of breast cancer increases with age doubling about every 10 years until the menopause, when the rate of increase slows dramatically. 4,5 This probably owes to diminishing level of circulating oestrogen. 4,6

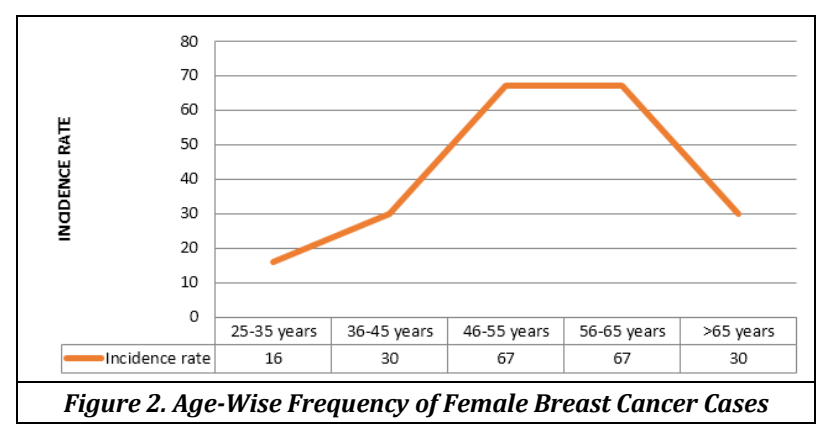

The graph we obtained after statistical analysis is similar to the age specific graph depicted elsewhere ${ }^{5,6}$ The slope of the curve after menopause may be flat, or even negative. This is a consequence of increasing risks of occurrence in consecutive generations of women rather than a real decline in risk with age. ${ }^{7}$ The young age structure of populations in developing countries (like ours) coupled with a rather flat age-incidence curve implies that mean age at diagnosis in developing countries is lower than that of European and American populations. ${ }^{6}$

In our study we found that Luminal-A was the most predominant type comprising about $43 \%$, followed by Triple negative and HER2 positive types which makes $21 \%$. Least dominant was the Luminal-B type which account for only $13 \%$ of all breast cancer types. A similar result was obtained by Wayne et al in his retrospective study of breast cancer patients. But it holds true only with respect to Luminal-A type. ${ }^{8}$ The order of frequency in general is Luminal-A, the most predominant followed by basal like followed by HER2 positive and Luminal-B. ${ }^{8}$

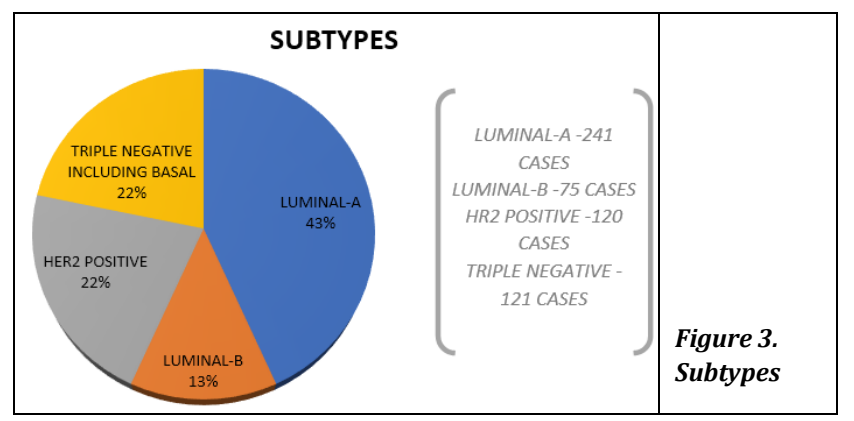

Earlier reports suggested that 3 biomarkers that are routinely used to asses breast cancers in clinical practice ie., ER, PR \& HER2 ${ }^{9}$ can be used to approximate the molecular category of breast cancer as defined by gene expression profiling. ${ }^{10}$ Now by using Ki-67 proliferation index, the luminal typing is based on its expression solely. ${ }^{11}$ As such we got 107 cases showing HER2 over-expression with 33 cases showing $3+$ positivity.

Luminal-B: In our study, luminal-b was the least frequent type. As said in the methodology it is a tumour having a high Ki67 index. They generally grow slightly faster than Luminal-A and their prognosis is slightly worse ${ }^{12}$. As in the study by Maggie et al, $19 \%$ of the cohort population showed Luminal-B, whereas in a larger sample of 2847 by the same investigator showed $7-10 \%$ luminal-B with $95 \%$ confidence interval. This is found to be in consensus with our study of 557 specimen.

In luminal-B subtype, irrespective of HER2 status, the high Ki-67 index characterizes it ${ }^{12}$. In our study, the HER2 positive and HER2 negative cases occurs in almost equal proportion.

\section{Triple Negative Cases (Including Basal-Like)}

As described earlier, the triple negative cases are those which show Hormone receptor negativity (ER-, PR-, HER2-).13 Among this triple negative category when the basal markers (CK5/6 and/or EGFR) are expressed, it is labelled as 'Basal-like'. Histopathologically these are high grade tumours, with a younger age patient, with a poor prognosis. ${ }^{14,15}$

In our study we had 121 Triple negative cases (Including basal like) out of the total 557 cases. It represents $21.7 \%$ of our cases. Among the 121 Triple negative tumours, 93 tumours expressed basal markers and so were included in 'Basal-like' tumours. The rest 23 tumours did not even express the basal markers making them negative for all markers (ER-, PR-, HER2, CK5/6-, EGFR-).

The best currently known panel of antibodies which is routinely used to diagnose the basal-like subtype of breast cancer has been proposed by Nielsen et al. It comprises 4 antibodies which are ER, HER2, CK5/6, and EGFR. This has shown to produce a $100 \%$ specificity and about $76 \%$ sensitivity. There are several basal like breast cancer (BLBC) biomarkers potentially useful in clinical diagnosis, but to date none of them has shown sufficiently high sensitivity and specificity to compete with the panel described above. By using the above said panel of antibodies, a tumour to be labelled as a 
'Basal-like' is still a matter of controversy. ${ }^{16}$ In literature it has been said that either (a) Lack of expression of ER \& HER2 in conjunction with expression of CK5/6 and/or EGFR 25 or (b) Lack of expression of ER, PR and HER2 in conjunction with expression of CK5/6 and/or EGFR. 17

In our study, of the 93 cases that showed 'Basal-like' phenotype, 84 cases showed expression of both the Basal markers (CK5/6 \& EGFR), 2 cases showed only CK5/6 expression and 7 cases showed only EGFR expression.

\section{Various Types of Breast Cancer}

In our study of 557 breast cancer, we were fortunate enough to get different types of breast cancer and thus had the privilege to study the different subtypes expressing in each. So, apart from the Invasive Ductal Carcinoma NOS, which is the most common variant, there was Invasive Lobular Carcinoma (Diagnosed by loss of expression of E-Cadherin), Mucinous Carcinoma, Tubular Carcinoma and IDC with DCIS component. Their proportion was $1.7 \%, 1.9 \%, 0.3 \%$ and $2.3 \%$ respectively. There was also one case each of Metaplastic carcinoma, Medullary carcinoma and Micropappilary carcinoma. The Metaplastic and Medullary Carcinoma showed a 'Basal-like' phenotype, whereas most of the Mucinous and Lobular Carcinoma were of the 'Luminal-A' type. 'Basal-like' tumours were indeed very rare in Mucinous \& Lobular Carcinoma.

\begin{tabular}{|c|c|c|}
\hline \multirow{2}{*}{ ILC } & Luminal-A & $60 \%$ \\
\cline { 2 - 3 } & HER2 & $30 \%$ \\
\cline { 2 - 3 } & Luminal-B & $10 \%$ \\
\hline \multirow{3}{*}{ Mucinous Carcinoma } & Luminal-A & $54.5 \%$ \\
\cline { 2 - 3 } & Luminal-B & $27.2 \%$ \\
\cline { 2 - 3 } & HER2 & $9 \%$ \\
\cline { 2 - 3 } & Basal Like & $9 \%$ \\
IDC with DCIS & Luminal-A & $46.15 \%$ \\
\cline { 2 - 3 } & Luminal-B & $30.7 \%$ \\
\cline { 2 - 3 } Tubular Carcinoma & HER2 & $23.1 \%$ \\
\cline { 2 - 3 } & Luminal-A & $50 \%$ \\
\hline Metaplastic Carcinoma & Basal Like & $100 \%$ \\
\hline Medullary Carcinoma & Basal Like & $100 \%$ \\
\hline \multirow{2}{*}{$\begin{array}{c}\text { Table 2. Various Subtypes as per the Panel of Surrogate IHC Markers } \\
\text { in Different Breast Cancer Types. The Proportion of Each Subtype is } \\
\text { also Noted }\end{array}$} \\
\hline
\end{tabular}

\section{DISCUSSION}

In our study of 557 cases, the most common surgery performed was Modified radical mastectomy which accounts for more than $50 \%$ of the whole specimen. It was followed by the trucut biopsy specimen which showed a $34 \%$ incidence. Even with the meagre tissue available in most of the trucut biopsy, we could do the complete panel of markers and thus finally subtype the breast cancers. This was particularly beneficial in-patient point of view as it would help them in commencing an earlier personalized treatment regime. Thus, will not only reduces the mortality, but also significantly decreases the morbidity of the unwanted radical surgeries.

\section{Male Breast Cancer}

The female to male incidence rate ratios (FMIRR) have been calculated across countries and found to be 122 in a retrospective study by Diana et al..$^{2}$ In our study we found it as 98 female breast cancer cases to 2 male breast cancer cases.
This we found to be more likely to go with the global trend. The study ${ }^{18}$ by Robert Kornegoor et al in 2014, showed $75 \%$ cases as Luminal-A, $21 \%$ cases as Luminal-B and least common were triple negative ${ }^{18}$ in male breast cancer. Our study also showed a very similar results with $78 \%$ showing luminal type and almost none showed a triple negative case. Since the male breast cancer is understudied like other rare "Orphan" diseases, the age specificity has also been underlooked ${ }^{3}$. But over the past 25 years the incidence has increase 18,3 and so also the age specificity. In our study we found the mean age of male breast cancer as 65 years which is in accordance with the study of Gregory et al that showed a median age of 68 in 71 male breast cancer cases. ${ }^{19}$

\section{Female Breast Cancer}

Age incidence curve across the world have shown a flat curve after attaining menopause. As with most epithelial tumours, the incidence of breast cancer increases rapidly with age. ${ }^{3,4}$ The incidence of breast cancer increases with age doubling about every 10 years until the menopause, when the rate of increase slows dramatically.4,5 This probably owes to diminishing level of circulating estrogen.4,6 The graph we obtained after statistical analysis is similar to the age specific graph depicted elsewhere.5,6 The slope of the curve after menopause may be flat, or even negative. This is a consequence of increasing risks of occurrence in consecutive generations of women rather than a real decline in risk with age. ${ }^{7}$ The young age structure of populations in developing countries (like ours) coupled with a rather flat age-incidence curve implies that mean age at diagnosis in developing countries is lower than that of European and American populations. ${ }^{6}$

\section{Types of Carcinoma Breast in Order of Frequency}

In our study we got the predominant type as Luminal-A. A similar result was obtained by Wayne et al in his retrospective study of breast cancer patients. But it holds true only with respect to Luminal-A type ${ }^{8}$. The order of frequency in general is Luminal-A, the most predominant followed by basal like followed by HER2 positive and Luminal-B. ${ }^{8}$

\section{ER \& PR Status}

ER positive and ER negative breast cancers are fundamentally distinct at transcriptomic level.4,7 It is estimated that every 2 out of 3 breast cancers are ER positive. ${ }^{20}$ It is of debate that PR status has a clinically effective outcome. Both oestrogen and progesterone receptors are present on the breast epithelial cells. The PR positivity shows that there is an intact oestrogen receptor pathway.4,21 Targeted chemotherapy will be given to the patient when the ER status becomes positive irrespective of the PR status. ${ }^{22}$ In our study we got 8 cases as ER negative but PR positive. The clinical importance of such a situation is not known. Since the literature says that existence of ER-/PR+ tumour is ambiguous, we did twice the IHC for ER and PR to confirm the results.

\section{HER2 Status}

In our study we had 120 cases of HER2 positive tumours which comprises about $21 \%$ of all types of breast carcinomas. This is in consensus with the study by Wayne et al which showed $3^{\text {rd }}$ most frequent sub type as HER2 positive. 


\section{KI-67 Proliferation Index \& Luminal Typing}

With the use of Ki-67 surrogate IHC marker for proliferation it is now easily possible to classify breast carcinoma into Luminal-A and Luminal-B.

\section{CONCLUSIONS}

This study was an attempt to subclassify the multifaceted breast carcinoma at molecular level using surrogate IHC markers. We used a panel of 6 biomarkers for better accuracy and precision as seen in previous studies. The 6 biomarkers used are ER, PR, HER2, CK5/6, EGFR, Ki-67. Our study is the first one of its kind to be carried out in Government Medical College, Thiruvananthapuram, and so also in our state Kerala. As this is the first study to be carried out in our college, there has been some ambiguous typing of a few breast carcinoma and the expression of some IHC markers that the literature does not show any evidence of its existence.

\section{REFERENCES}

[1] Andrewson WF, Jatoi I, Tse J,et al. Male Breast Cancer: a population based comparison with Female breast cancer. National Cancer Centre and National Institute of Health. Journal of Clin Oncology 2010;28(2):232-9.

[2] Ly D, Forman D, Ferlay J, et al. An Institutional comparison of male and female breast cancer incidence rates. Int J Cancer 2013;132(8):1918-26.

[3] Giordano SH. A review of the diagnosis and management of male breast cancer. The Oncologist 2005;10(7):471-9.

[4] WHO Classification of Tumours of breast - 2017 edn.

[5] McPherson K, Steel CM, Dixon JM. ABC of breast diseases. Breast cancer - epidemiology, risk factors and genetics. BMJ 2000;321(7261):624-8.

[6] Bray F, McCarron P, Parkin DM. The changing global patterns of female breast cancer incidence and mortality. Breast Cancer Res 2004;6(6):229-39.

[7] Henderrom BE, Ross R, Bernstein L. Estrogens as a cause of human cancer: the Richard and Hinda Rosenthal Foundation award lecture. Cancer Res 1988;48(2):246-53.

[8] Ihemelandu CU, Leffall LD Jr, Dewitty RL, et al. Molecular breast cancer subtypes in pre-menopausal and postmenopausal African-American women: age specific prevalence and survival. Journal of Surgical Research 2007;143(1):109-18.
[9] Cheang MCU, Chia SK, Voduc D, et al. Ki67 index HER2 status and prognosis of patients with luminal B breast cancer. Journal National Cancer Institute 2009;101(10):736-50.

[10] Schnitt SJ. Classification of prognosis of invasive breast cancer: from morphology to molecular taxonomy. Mod Pathol 2010;(23 Suppl 2):S60-4.

[11] Ge Y, Sneige N, Eltorky MA, et al. Immunohistochemical characterization of subtypes of male breast carcinoma. Breast Cancer Research 2009;11(3):R28.

[12] Ciocca V, Bombonati A, Gatalica Z, et al, Cytokeratin profiles of male breast cancers. Histopathology 2006;49(4):365-70.

[13] Badowska-Kozakiewicz AM, Budzik MP. Immunohistochemical characteristics of basal-like breast cancer. Contemp Oncol (Pozn) 2016;20(6):436-43.

[14] Nielsen TO, Hsu FD, Jensen K, et al, Immunohistochemical and clinical characterization of the basal-like subtype of invasive breast carcinoma. Clin Cancer Res 2004;10(16):5367-74.

[15] Badve S, Dabbs DJ, Schnitt SJ, et al. Basal-like and triplenegative breast cancers: a critical review with an emphasis on the implications for pathologists and oncologists. Modern Pathology 2011;24(2):157-67.

[16] Bombonati A, Sgroi DC. The molecular pathology of breast cancer progression. J Pathol 2011;223(2):307-17.

[17] Geyer FC, Rodrigues DN, Weigelt B, et al. Molecular classification of estrogen receptor-positive/luminal breast cancers. Adv Anat Pathol 2012;19(1):39-53.

[18] Kornegoor R, Verschuur-Maes AH, Buerger $H$, et al. Molecular subtyping of male breast cancer by immunohistochemistry. Modern Pathology 2012;25(3):398-404.

[19] Evans GF, Anthony T, Turnage RH, et al. The diagnostic accuracy of mammography in the evolution of male breast disease. American Journal of Surgery 2001;181(2):96100.

[20] Hefti MM, Hu R, Knoblauch NW, et al. Estrogen receptor negative/ progesterone receptor positive breast cancer is not a reproductive subtype. Breast Cancer Res 2013;15(4):R68.

[21] Hetchen J. Her2 Positive breast cancer, causes, symptoms and statistics. WebMD 2017.

[22] Neilsen TO, Hsu FD, Jensen K, et al. Immunohistochemical and clinical characterisation of the basal like subtype of invasive breast carcinoma. Clinical Cancer Research J 2004;10(16):5367-74. 